\title{
The Discovery of lambda Bootis Stars - The Southern Survey II
}

\author{
Simon J. Murphy ${ }^{1,2 \star}$, Richard O. Gray ${ }^{3}$, Christopher J. Corbally ${ }^{4}$, \\ Charles Kuehn ${ }^{1,5}$ Timothy R. Bedding ${ }^{1,2}$ and Josiah Killam ${ }^{3}$ \\ 1 Sydney Institute for Astronomy (SIfA), School of Physics, University of Sydney, NSW 2006, Australia \\ 2 Stellar Astrophysics Centre, Department of Physics and Astronomy, Aarhus University, 8000 Aarhus C, Denmark \\ 3 Department of Physics and Astronomy, Appalachian State University, Boone, NC 26808, USA \\ ${ }^{4}$ Vatican Observatory Research Group, Steward Observatory, Tucson, AZ, 85721-0065 USA \\ 5 Department of Physics and Astronomy, University of Northern Colorado, Greeley, CO 80639, USA
}

Accepted XXX. Received YYY; in original form ZZZ

\begin{abstract}
The $\lambda$ Boo stars are chemically peculiar A-type stars whose abundance anomalies are associated with the accretion of metal-poor material. We searched for $\lambda$ Boo stars in the southern hemisphere in a targeted spectroscopic survey of metal-weak and emissionline stars. Obtaining spectra for 308 stars and classifying them on the MK system, we found or co-discovered 24 new $\lambda$ Boo stars. We also revised the classifications of 11 known $\lambda$ Boo stars, one of which turned out to be a chemically normal rapid rotator. We show that stars previously classified in the literature as blue horizontal branch stars or emission-line A stars have a high probability of being $\lambda$ Boo stars, although this conclusion is based on small-number statistics. Using WISE infrared fluxes, we searched our targets for infrared excesses that might be attributable to protoplanetary or debris discs as the source of the accreted material. Of the $34 \lambda$ Boo stars in our sample, 21 at various main-sequence ages have infrared excesses, confirming that not all $\lambda$ Boo stars are young.
\end{abstract}

Key words: stars: chemically peculiar - stars: circumstellar matter - stars: emissionline, Be - stars: early-type - stars: evolution

\section{INTRODUCTION}

Long-standing puzzles in astrophysics often contain clues on physics that is missing from stellar models. The $\lambda$ Boo stars are one such puzzle. They are chemically peculiar A or Ftypes stars first identified as a distinct class in the 1950s (Slettebak 1952, 1954), and a complete explanation for their peculiarity is still lacking despite recent efforts (Jura 2015; Kama et al. 2015; Jermyn \& Kama 2018). They are characterised by metal weaknesses with a specific chemical abundance profile. Refractory elements such as magnesium and iron-peak elements are underabundant by -0.5 to -2.0 dex (Andrievsky et al. 2002) while volatile elements such as carbon, nitrogen, and oxygen have near-solar abundances (Baschek \& Slettebak 1988; Kamp et al. 2001; Folsom et al. 2012).

The abundance dichotomy between refractories and volatiles suggests that accretion from a circumstellar disc

^ E-mail: simon.murphy@sydney.edu.au (SJM) plays a role in the development or maintenance of the chemical anomalies (Venn \& Lambert 1990; Turcotte \& Charbonneau 1993; King 1994). The material itself does not need to be metal-weak because a variety of efficient dust-gas separation mechanisms can operate around A stars (Jermyn \& Kama 2018), allowing volatile-rich gas to be accreted onto the star without the refractory dust (Waters et al. 1992). Suggestions for the accretion source have included material left-over from star formation (Holweger \& Sturenburg 1993), gas from dense regions of the ISM (Kamp \& Paunzen 2002), and material ablated from hot jupiters (Jura 2015). Protoplanetary discs are particularly likely sources, especially since embedded planets can deplete the dust in a way that reproduces observed $\lambda$ Boo abundances (Kama et al. 2015; Jermyn \& Kama 2018). VLTI and ALMA observations confirm the existence of planets embedded in the discs of some $\lambda$ Boo stars (e.g. Matter et al. 2016; Fedele et al. 2017; Cugno et al. 2019; Toci et al. 2020).

Numerical calculations have shown that peculiarities from selective accretion ought to persist for only $10^{6} \mathrm{yr}$ once 
accretion has stopped (Turcotte \& Charbonneau 1993), before particle transport processes erase the chemical abundance signature. It then follows that most $\lambda$ Boo stars should be actively accreting. However, Gray et al. (2017) found that $\lambda$ Boo stars were no more likely to be observed with a debris disc at $22 \mu \mathrm{m}$ than chemically normal A stars. It is also apparent that not all $\lambda$ Boo stars are young: they are found at a wide range of main-sequence ages when placed on an HR diagram, according to either their spectroscopic $\log g$ values (Iliev \& Barzova 1995) or luminosities derived from precise Gaia parallaxes Murphy \& Paunzen (2017).

The age range of $\lambda$ Boo stars suggests a reservoir of material may be needed from which the star can accrete at an arbitrary age. Such a reservoir may include comets, such as the 400-Earth-mass cloud of CO-rich comets postulated to orbit the A stars HD 21997 and 49 Cet (Zuckerman \& Song 2012). So-called 'swarms' of comets, not unlike the fragmented comet Shoemaker-Levy 9 that delivered large quantities of volatiles to Jupiter (Lellouch et al. 1997), have been used to explain the peculiar transits of the Kepler A star KIC 8462852 (Boyajian et al. 2016; Bodman \& Quillen 2016). Such bodies, sometimes called Falling Evaporating Bodies (FEBs), may be perturbed from dormant orbits by mean-motion resonances with massive planets (Freistetter et al. 2007) or encounters with nearby stars (Bailer-Jones 2015; Gray et al. 2017). This comet-reservoir scenario has a pedigree in $\beta$ Pic (King \& Patten 1992; Gray \& Corbally 2002), a planet-host with $\lambda$ Boo-like properties (Lagrange et al. 2010; Cheng et al. 2016; Snellen \& Brown 2018), FEBlike spectral absorption signatures (Ferlet et al. 1987; Karmann et al. 2001; Thébault \& Beust 2001; Karmann et al. 2003), and transiting exocomets (Zieba et al. 2019).

A solution to the $\lambda$ Boo puzzle requires a broad approach, including particle transport models for stars and discs, and a larger and better characterised set of observations. To address the latter, Murphy et al. (2015) reinvestigated all known and candidate $\lambda$ Boo stars to create a homogeneous catalogue of class members, resulting in 64 bona-fide $\lambda$ Boo stars and 45 candidates for which more observations are required for a definite classification. Since $\lambda$ Boo stars are rare, with only $2 \%$ of A stars belonging in the class (Gray \& Corbally 1998), further expansion of the membership list requires efficient target selection.

In Gray et al. (2017, Paper I, hereafter), we began a search for new $\lambda$ Boo stars using GALEX photometry to target A stars with UV excesses. The $\lambda$ Boo stars have reduced line blanketing in the UV because they are metal weak, and hence show UV excesses compared to normal stars. We found 33 new southern $\lambda$ Boo stars and confirmed 12 others with that approach. By modelling their spectral energy distributions, we were also able to search for infrared excesses to make an unbiased assessment of the occurrence rate of discs around $\lambda$ Boo stars, finding the aforementioned result that discs are no more likely around $\lambda$ Boo stars at $22 \mu \mathrm{m}$ than around normal stars.

This is the second paper in the series, also focussing on the southern hemisphere (declination $<+15 \mathrm{deg}$ ). Observations of northern targets are ongoing and will be presented in future papers. In this paper, we particularly target known emission-line stars. Folsom et al. (2012) observed that many emission-line A stars were also $\lambda$ Boo stars - an observation compatible with the hypothesis that $\lambda$ Boo stars are active accretors. In addition to the emission-line stars, we created a target list of metal-weak objects by examining their Strömgren photometry. Our target selection, observations, and spectral classification procedures are described in Sec. 2.

We also look for infrared excesses around our targets, which might indicate the source of the accreted material if the accretion episode is recent or ongoing. We describe our SED modelling and search for infrared excesses in Sec. 3, and present conclusions in Sec. 4 .

\section{METHOD}

\subsection{Sample selection}

To improve the success rate of searching for $\lambda$ Boo stars beyond the $2 \%$ one expects at random, we compiled a target list from several types of stars that we considered likely to yield new $\lambda$ Boo stars. Unlike Paper I, our goal was only to find more $\lambda$ Boo stars, and although we didn't specifically favour stars with infrared excesses, we did not actively eliminate such survey bias. A major focus in this work was emission-line A stars, but relatively few $(<50)$ of these are known. Blue horizontal branch (BHB) stars are another class of rare metal-weak A stars that we considered to be promising targets, since some might have been misclassified in the literature. The target list therefore contained a mixture of emission-line stars, BHB stars, and a large number of metal-poor stars selected using Strömgren photometry. Due to good weather and efficient observing, we added a further group of targets on the final night of the observing run, comprising A and early F stars observed in Campaign 01 of the K2 Mission. Targets were organised into groups based on how they were selected (see Table 1 ):

Group 0. Known $\lambda$ Boo stars. In order to verify that the spectra were suitable for accurate classification, we obtained spectra of eleven known $\lambda$ Boo stars, chosen according to availability on the sky at the time of observation. One of these, HD 111164, was classified as a $\lambda$ Boo star by Abt \& Morrell (1995), but turned out to be a chemically normal rapid rotator.

Group 1. Emission-line A stars (i). We used the criteria search function of the SIMBAD database (Wenger et al. $2000)$ to select spectral types matching "A[0-9]*e", where '[0-9]' represents any integer in this range, the asterisk is a wildcard of any length, and ' $\mathrm{e}$ ' is the standard notation for emission lines. These are Herbig Ae/Be stars (Herbig 1960; Hillenbrand et al. 1992), the hotter analogues of T Tauri stars (Joy 1945; Appenzeller \& Mundt 1989). We expected that focussing on emission line stars would increase the efficiency of our $\lambda$ Boo search by preferentially observing stars with circumstellar discs, or stars accreting material from an unknown source. Having a larger sample of such stars is also useful for ascertaining any link between age, accretion, and $\lambda$ Boo peculiarity. Of the 308 stars observed, 20 stars came from this group.

Group 2. Emission-line A stars (ii). This group is phenomenologically identical to the previous group, except that the search terms were slightly modified to capture stars whose spectral types had been recorded differently. We searched for object types matching 
"Em*/Ae*" and "spectral type = A". Of the 308 stars observed, 18 stars came from this group.

Group 3. Photometrically metal-weak stars. Strömgren photometry can be used quite efficiently to select metal-weak stars from a sample of A stars. The $m_{1}$ index is sensitive to metallicity, with metal-weak stars having lower values of $m_{1}$ than normal stars at a given $b-y$ colour (see Paunzen \& Gray 1997). We selected stars using the following criteria:

$$
\begin{aligned}
& \text { i. }-0.015<(b-y)<0.30 \\
& \text { ii. } m_{1}>0.130-0.3(b-y) \\
& \text { iii. } m_{1}<0.220-0.3(b-y) \\
& \text { iv. } c_{1}<1.4-2.0(b-y)
\end{aligned}
$$

and prioritised targets with Tycho $B$ magnitudes $<10$ that had not already been observed by Paunzen \& Gray (1997) or other papers in that series (Paunzen et al. 2001; Paunzen 2001). Of the 308 stars observed, 210 stars came from this group.

Group 4. Blue-horizontal-branch (BHB) stars. At classification resolution $(R \sim 3000)$, the spectra of BHB stars are quite similar to $\lambda$ Boo stars. We observed seven BHB stars from MacConnell et al. (1971) as a likely source of additional $\lambda$ Boo stars.

Group K2. Targets scheduled to be observed in Campaign 01 of the K2 Mission. Space photometry can be beneficial to the study of $\lambda$ Boo stars in multiple ways. For instance, there are $\lambda$ Boo stars with exoplanets, such as HR 8799 (Soummer et al. 2011), so space photometry might reveal exoplanet (or exocomet) transits around $\lambda$ Boo stars. In addition, the same photometry can be used for asteroseismology. Stellar oscillations are sensitive to metallicity, and can be used to determine whether stars are globally metal-poor or just have surface peculiarities (Murphy et al. 2013). We therefore observed some A-type stars that were scheduled to be observed in Campaign 01 of the K2 Mission (Howell et al. 2014). This group was not selected according to spectroscopic or photometric properties, so it is numbered differently from the others. It is also not anticipated to yield a higher number of $\lambda$ Boo stars than the $2 \%$ expected from a random draw of field stars. Of the 308 stars observed, 42 stars came from this group, and we found one (HD 98069) to be a $\lambda$ Boo star. Its K2 light curve reveals it is a $\delta$ Sct star with eight pulsation peaks exceeding $1 \mathrm{mmag}$ and a further seven exceeding $0.5 \mathrm{mmag}$, most of which lie between 12 and $18 \mathrm{~d}^{-1}$. Further asteroseismic analysis is beyond the scope of this work. A TESS light-curve is also available, has similar properties, and has been analysed along with the lightcurves of all southern $\lambda$ Boo stars by Murphy et al. (2020).

Our target list reflects our single-site, single-epoch observations (Sec. 2.2): only targets observable during 2014 Mar were included, corresponding roughly to right ascension in the range $75-300^{\circ}$. Our focus on emission line stars (Groups 1 and 2) produced many new targets not already searched for $\lambda$ Boo stars, whereas the Strömgren targets (Group 3) have an overlap of 21 targets with Paper I, which were observed at SAAO in 2013 and 2014. Some overlap is desirable to check for consistency between different instruments, noting of course that some targets may be spectrum variables. Because some of those 21 overlapping stars are $\lambda$ Boo stars, they are co-discoveries. Two stars (HD 94326 and HD 102541) whose SAAO spectra showed $\lambda$ Boo spec-
Table 1. Breakdown of the target selection groups described in Sec. 2.1, the number of stars in each group ultimately classified as $\lambda$ Boo stars (including the two uncertain " $\lambda$ Boo?" stars), the total number of targets in each group, and the percentage of $\lambda$ Boo stars obtained by dividing the previous two columns.

\begin{tabular}{ccrrr}
\hline Group & Description & Num. $\lambda$ Boo & Total & Percent \\
\hline 0 & Known $\lambda$ Boo stars & 10 & 11 & $91 \%$ \\
1 & "A $[0-9]^{*}$ e" & 4 & 20 & $20 \%$ \\
2 & "Em*/Ae*" and "A" & 1 & 18 & $6 \%$ \\
3 & Photometrically met wk & 16 & 210 & $8 \%$ \\
4 & BHB stars & 2 & 7 & $29 \%$ \\
K2 & K2 targets & 1 & 42 & $2 \%$ \\
\hline
\end{tabular}

tral features are classified as non- $\lambda$ Boo metal-weak stars in this work. More spectra and an abundance analysis are desirable to confirm whether these are indeed $\lambda$ Boo stars, and to analyse the variability in their spectra. Other than the 21 overlapping targets and the 11 in Group 0, the remainder (276) were unique to this survey.

\subsection{Observations}

During 2014 Mar 17-19 we obtained spectra of 308 targets with the WiFeS spectrograph (Dopita et al. 2007) on the ANU 2.3-m telescope at Siding Spring Observatory. Our spectra were obtained in the blue-violet region in B3000 mode and have a resolution of about $2.5 \AA / 2$ pixels. The $\mathrm{WiFeS}$ data were reduced with the PYWIFES software package (Childress et al. 2014). Due to difficulty in rectifying the spectra over the Balmer jump, we trimmed the spectra to the range $3865-4960 \AA$. The spectra thus cover the region between the blue wing of $\mathrm{H} 8$ and the red wing of $\mathrm{H} \beta$. The spectra are qualitatively similar to those made from SAAO for Paper I.

\subsection{Spectral classification}

We classified the spectra on the MK system, which is described by Gray \& Corbally (2009). The $\lambda$ Boo stars are described in detail there and in Paper I, so we give only a summary here. When classifying A stars, the three main temperature criteria are (i) the strength of the Ca II K line, which rapidly increases towards later (cooler) types; (ii) the strength of the Balmer lines of hydrogen, which have a broad maximum around A2 and decrease on either side; and (iii) the metal lines, which increase in strength almost uniformly from A0 to F0. Ordinarily, all of these are absorption lines and in a normal star, all three criteria would yield the same temperature subclass. This is not the case in the $\lambda$ Boo stars, where the metal lines are weak for a given hydrogen line type. It is the hydrogen lines that give the best estimate of the true stellar temperature, hence the spectra are usually classified with their hydrogen line type, then the luminosity class, then the $\mathrm{K}$ and metal line types, e.g. A7 V kA2mA2 $\lambda$ Boo. Spectral types of $\lambda$ Boo stars having only mild peculiarity are written with the class name in parentheses: "( $\lambda$ Boo $) "$. For F-type stars, the G-band becomes an important feature, and this is sometimes written prepended with a 'g', e.g. F5 V mF2gF5.

Each spectrum was classified by SJM and independently 
by at least one other author (ROG or CJC), and without knowledge of which group the target originated from. Any spectrum for which the initial classifications were found to disagree was reclassified by all three classifiers and discussed until agreement was reached on the best-fitting spectral type. The spectral types of the targets are given in Table A1. For explanations of notation used in spectral classes, e.g. 'e' for emission and 's' for sharp-lined, see Gray \& Corbally (2009) and Smith et al. (2011).

\section{STELLAR PARAMETERS AND INFRARED EXCESSES}

Establishing the mechanisms that lead to $\lambda$ Boo peculiarities requires a better understanding of the environments of the stars. In particular, the accretion of dust-depleted material requires a reservoir, whose thermal emission might be detectable above the stellar luminosity in the infrared. To search for this, we constructed spectral energy distributions (SEDs) of our targets using stellar atmosphere models, against which we compared infrared fluxes from 2MASS and WISE. We followed the method from Paper I, which is summarised in this section.

\subsection{Physical Parameters and Reddening}

Stellar physical parameters were determined via $\chi^{2}$ minimisation between the observed spectra and a library of synthetic spectra computed with SPECTRUM (Gray \& Corbally 1994) and ATLAS9 (Castelli \& Kurucz 2004). The library grid has effective temperatures spanning 6500$25000 \mathrm{~K}$ (having $50-\mathrm{K}$ spacing up to $10000 \mathrm{~K}$, then $100-\mathrm{K}$ spacing to $11500 \mathrm{~K}, 500-\mathrm{K}$ spacing to $13000 \mathrm{~K}$, and $1000-$ $\mathrm{K}$ spacing to $25000 \mathrm{~K}$ ), with $\log \mathrm{g}=3.3,3.6,4.0$, and 4.2 , and with metallicities of $[\mathrm{M} / \mathrm{H}]=+0.5,+0.2,0.0,-0.2,-0.5$, $-1.0,-1.5$, and -2.0 . We used the stellar spectral types (see Sec. 2.3) to estimate the intrinsic $(B-V)_{0}$ colours of the stars according to the relation in Paper I, making allowances for differences in stellar metallicity.

Photometric fluxes were downloaded from IPAC. ${ }^{1}$ We used Johnson B and V; 2MASS J, H and $\mathrm{K}$; and WISE $\mathrm{W} 1, \mathrm{~W} 2, \mathrm{~W} 3$ and $\mathrm{W} 4$. Reddening $(E(B-V))$ was evaluated by comparing the observed $B-V$ colours with our intrinsic $(B-V)_{0}$ colours, and the infrared fluxes were dereddened with a combination of the Fitzpatrick reddening law (Fitzpatrick 1999) and the mid-infrared extinction law of Xue et al. (2016). When Johnson B and V were unavailable, we used Tycho $\mathrm{B}_{\mathrm{T}}$ and $\mathrm{V}_{\mathrm{T}}$ (Høg et al. 2000) instead and followed a similar reddening procedure with a slightly different relation (Paper I) to account for the difference in zero-points of the two photometric systems (Bessell \& Murphy 2012).

\subsection{Infrared Excesses}

We compared the W1, W2, W3 and W4 fluxes to the synthetic spectra to identify stars with infrared excesses. We normalised the spectra to the 2MASS J band, except where there were clear excesses in the 2MASS bands, in which

\footnotetext{
1 https://irsa.ipac.caltech.edu/
}

case spectra were normalised to the $\mathrm{V}$ band instead. We recorded infrared excesses (in W1-W4) in the form of a flux ratio, $\left(F_{\text {obs }}-F_{\text {model }}\right) / F_{\text {model }}$, and calculated the significance of those excesses using the recorded errors for the WISE photometry. Following Paper I, we considered infrared excesses significant at $2 \sigma$ rather than the conventional $3 \sigma$, to avoid missing potentially interesting targets for future follow-up. This is particularly important for the detection of cool discs that do not radiate strongly at wavelengths below $22 \mu \mathrm{m}$ (i.e. WISE W4). Stars with excesses at $\geq 2 \sigma$ are indicated in Table A1, and the values and significances of the excesses are given in Table 2. SED parameters for stars without infrared excesses are given separately in Table 3 .

We find that 21 of the $34 \lambda$ Boo stars in our sample have IR excesses. Seven of them exceed $10 \sigma$ in strength, and six of those (HD 101412, HD 139614, HD 141569, HD 169142, NGC 638322 , and T Ori) have excesses that are larger at longer wavelengths, suggesting circumstellar discs (Fig.1). We found emission lines in the spectrum of HD 139614, which is known to be a pre-main sequence star with a protoplanetary disc (Matter et al. 2016; Carmona et al. 2017; Laws et al. 2020), in the less well-studied accretor HD 101412 (Cowley et al. 2012; Schöller et al. 2016), and in the cluster member T Ori. For HD 141569 and HD 169142, we found no emission in our spectra, even though HD 141569 is known to have a Kuiper-Belt-like debris disc (Mendigutía et al. 2017; Mawet et al. 2017; Miley et al. 2018; White et al. 2018; Bruzzone et al. 2020) and HD 169142 has a protoplanetary disc (Fedele et al. 2017; Carney et al. 2018; Ligi et al. 2018; Chen et al. 2019; Gratton et al. 2019; Macías et al. 2019; Toci et al. 2020). For NGC 638322 , our spectrum shows weak emission. Further observations of this target would be worthwhile, especially high-resolution spectroscopy in the visible for an abundance analysis, and ALMA or VLT observations for dust characterisation. The seventh target with a $>10 \sigma$ IR excess is HD 314915. Although this is classified as an emission-line star on SIMBAD (from Nesterov et al. 1995), its SED appears to be more consistent with a cool binary companion (Fig. 2).

Table 4 shows the fraction of stars in each target selection group with infrared excesses. The K2 targets constitute the only group that is presumably unbiased with respect to infrared excess, and in that group, out of 41 normal A-type stars, 10 show excesses at $\geq 2 \sigma$ in one or more WISE bands. That is a proportion of $24.4 \pm 7.7 \%$. In Paper I, 18 out of 121 normal A-type stars in the Tycho sample showed excesses, giving a proportion of $14.9 \pm 3.5 \%$. According to a two-tailed $\mathrm{Z}$ test, the resulting $\mathrm{z}$-score is 1.3225 , with a $\mathrm{p}$ value of 0.187 , so those two proportions are not significantly different. Combining the K2 and Tycho normal star samples, we find that out of a total of 162 normal A-type stars, 28 show WISE $2 \sigma$ excesses, or a proportion of $17.3 \pm 3.3 \%$. This is similar to the $20.0 \pm 10 \%$ observed for $\lambda$ Boo stars in Paper I, although a larger unbiased sample of $\lambda$ Boo stars is clearly needed before we can make any meaningful statement about whether the proportion of $\lambda$ Boo stars with IR excesses differs from that of normal A-type stars.

\subsection{Luminosities}

To position the $\lambda$ Boo stars in our sample on the HR diagram, we determined their luminosities. We followed the 

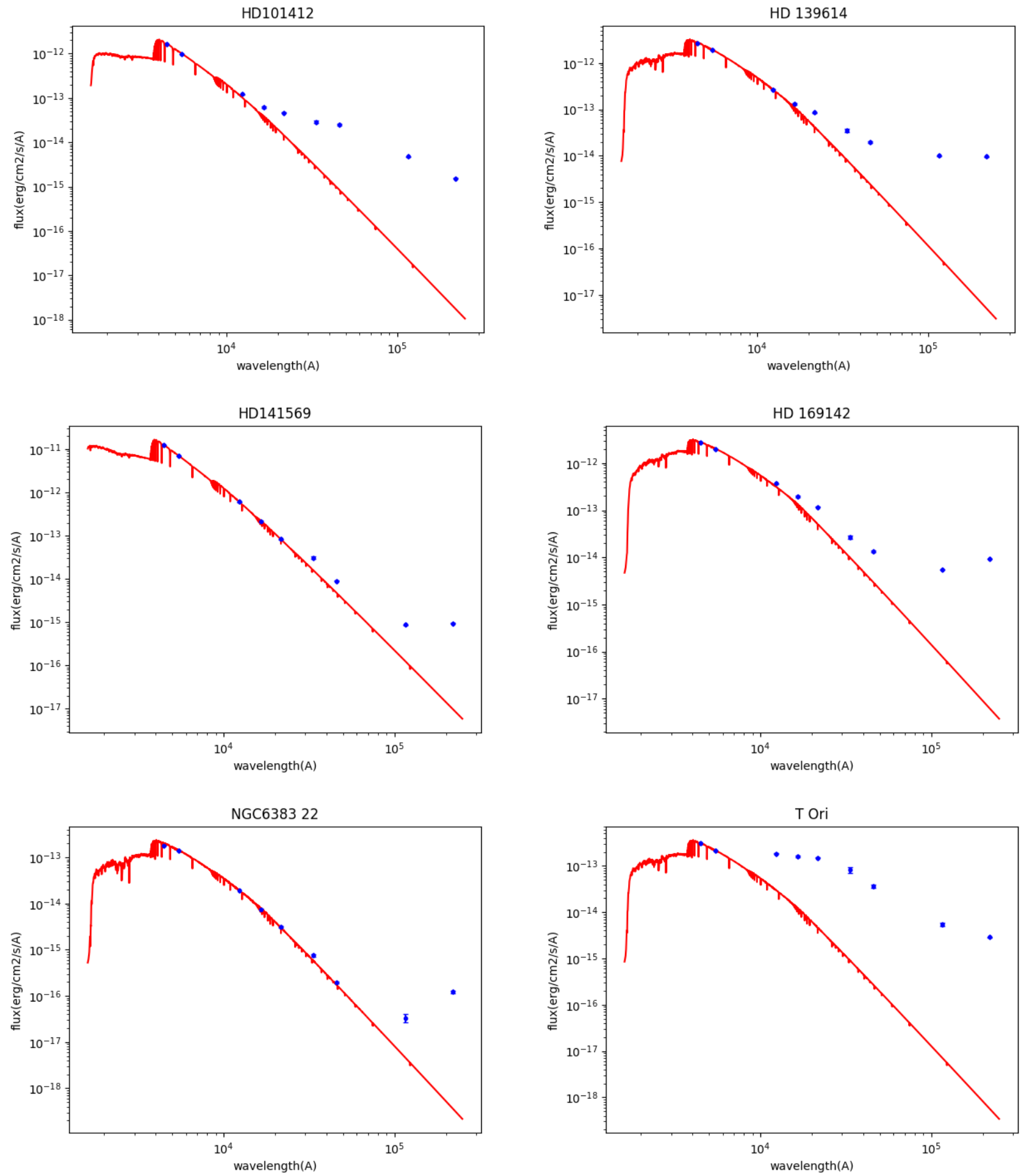

Figure 1. Spectral energy distributions (SEDs) for the six $\lambda$ Boo stars with strong infrared excesses ( $>10 \sigma$ in any of the WISE passbands) that probably originate from discs. Blue data points are photometric fluxes in Johnson B and V; 2MASS J, H and K; and WISE W1, $\mathrm{W} 2$, W3 and W4.

methodology of Murphy et al. (2019) and Hey et al. (2019), except that we used the Johnson $\mathrm{V}$ band rather than SDSS g. Bolometric luminosities were calculated via absolute magnitudes using standard formulae:

$M_{V}=m_{V}-5(\log d-1)-A_{V}$ and

$\log L_{\mathrm{bol}} / L_{\odot}=-\left(M_{V}+B C-M_{\mathrm{bol}, \odot}\right) / 2.5$.

The apparent $\mathrm{V}$ magnitudes, $m_{V}$, are those in Table A1, which are taken from the SIMBAD database with an assumed uncertainty of $0.02 \mathrm{mag}$. The V-band extinctions, $A_{V}$, were taken as $3.1 E(B-V)$, using the $E(B-V)$ values deter- 
Table 2. Infrared excesses for all stars of the sample with a $\geq 2 \sigma$ excess in one or more WISE bands. Twelve rows are shown; the full table is available online in machine-readable format. Model parameters $\left(T_{\text {eff }}, \log g,[\mathrm{Fe} / \mathrm{H}]\right.$ and $\left.E(B-V)\right)$ describe the spectral energy distributions, and asterisks in the $T_{\text {eff }}$ column indicate the stars for which the $V$ band rather than the $J$ band was used for normalisation. Infrared excesses ("val.") are given as the flux fraction in excess of the model, i.e. $\left(F_{\text {obs }}-F_{\text {model }}\right) / F_{\text {model }}$.

\begin{tabular}{|c|c|c|c|c|c|c|c|c|c|c|c|c|c|}
\hline \multirow[t]{2}{*}{ Obj. Name } & \multirow[t]{2}{*}{ Spectral Type } & \multirow{2}{*}{$\begin{array}{r}T_{\text {eff }} \\
\mathrm{K}\end{array}$} & \multirow[t]{2}{*}{$\log g$} & \multirow[t]{2}{*}[\mathrm{Fe}/\mathrm{H}]{} & \multirow{2}{*}{$\begin{array}{r}E(B-V) \\
\text { mag }\end{array}$} & \multicolumn{2}{|c|}{ W1 } & \multicolumn{2}{|c|}{ W2 } & \multicolumn{2}{|c|}{ W3 } & \multicolumn{2}{|c|}{ W4 } \\
\hline & & & & & & val. & $\bar{\sigma}$ & val. & $\bar{\sigma}$ & val. & $\bar{\sigma}$ & val. & $\sigma$ \\
\hline BD-15 1548 & B7 IIIe He-wk & $13000 *$ & 3.3 & 0.0 & 0.2 & 1.2 & 25.8 & 1.5 & 32.9 & 3.5 & 40.7 & 9.5 & 10.1 \\
\hline BD-15 4515 & $\mathrm{~F} 2 \mathrm{VkA} 4 \mathrm{~mA} 6 \lambda \mathrm{Boo}$ & 7000 & 4.2 & -1.5 & 0.108 & 0.1 & 3.6 & 0.1 & 5.5 & 0.1 & 6.0 & $\ldots$ & $\ldots$ \\
\hline CD-37 3833 & $\mathrm{~A} 2 \mathrm{Vn} \mathrm{kA} 0$ & 8700 & 4.2 & -0.5 & 0.05 & $\ldots$ & $\ldots$ & $\ldots$ & $\ldots$ & 0.1 & 2.4 & $\ldots$ & $\ldots$ \\
\hline CD-48 3541 & $\mathrm{~A} 2 \mathrm{Vn} \mathrm{kA} 0 \mathrm{~mA} 1$ & $8900 *$ & 4.2 & -1.0 & 0.04 & $\ldots$ & $\ldots$ & $\ldots$ & $\ldots$ & 0.1 & 2.1 & 1.5 & 2.7 \\
\hline CD-55 2595 & $\mathrm{~B} 1 \mathrm{Ve}$ & $25000 *$ & 4.0 & 0.0 & 0.31 & 0.3 & 12.1 & 0.6 & 20.9 & 1.4 & 19.9 & $\ldots$ & $\ldots$ \\
\hline CD-59 1764 & $\mathrm{~A} 0.5 \mathrm{~V}$ & 9650 & 4.2 & 0.0 & 0.052 & $\ldots$ & $\ldots$ & $\ldots$ & $\ldots$ & 0.1 & 3.4 & 1.0 & 2.1 \\
\hline CD-60 4157 & A1 Van & 9500 & 4.2 & 0.0 & 0.162 & $\ldots$ & $\ldots$ & $\ldots$ & $\ldots$ & $\ldots$ & $\ldots$ & 1.0 & 3.3 \\
\hline CPD-58 3138 & $\mathrm{~A} 1.5 \mathrm{Vs}$ & 9200 & 4.2 & 0.0 & 0.06 & 0.1 & 2.8 & 0.0 & 2.4 & $\ldots$ & $\ldots$ & $\ldots$ & $\ldots$ \\
\hline HD 100380 & $\mathrm{~A} 4 \mathrm{IVs}$ & 8350 & 4.0 & 0.0 & 0.035 & $\ldots$ & $\ldots$ & 0.1 & 3.2 & $\ldots$ & $\ldots$ & 0.1 & 3.4 \\
\hline HD 100453 & F1 Vn & $7100 *$ & 4.2 & 0.0 & 0.0 & 7.1 & 4.0 & 20.9 & 5.3 & 184.4 & 98.7 & 2283.8 & 136.2 \\
\hline HD 101412 & $\mathrm{~A} 3 \mathrm{~V}(\mathrm{e}) \mathrm{kA} 0.5 \mathrm{~mA} 0.5(\lambda \mathrm{Boo})$ & $8500 *$ & 4.2 & -1.5 & 0.114 & 9.2 & 16.0 & 28.6 & 18.0 & 208.8 & 108.6 & 888.1 & 90.9 \\
\hline
\end{tabular}

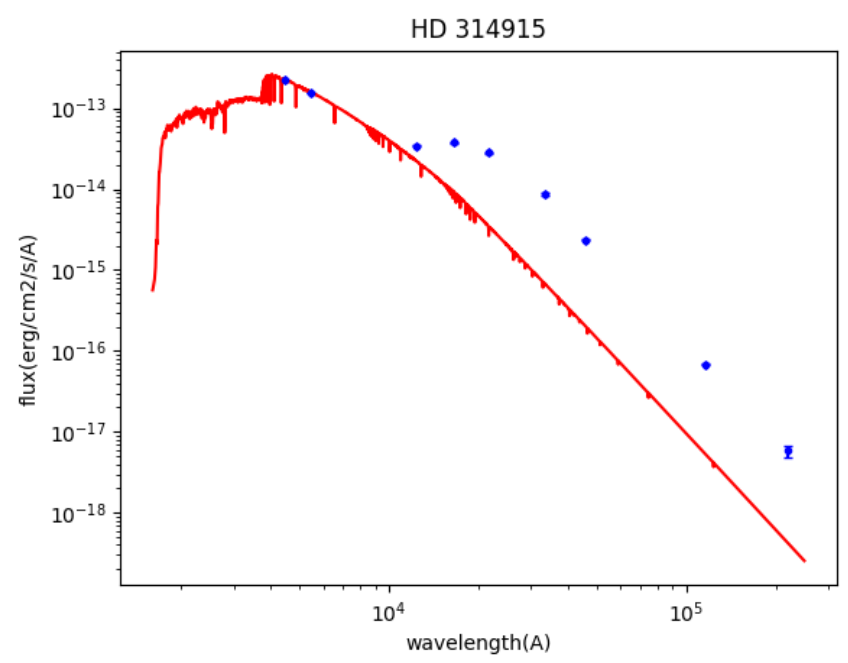

Figure 2. The SED of the $\lambda$ Boo star HD 314915. Unlike the dischosts whose SEDs are shown in Fig. 1, this infrared excess is more consistent with a cooler stellar companion.

Table 3. Parameters from SED fitting, for the stars without detected infrared excesses. Asterisks in the $T_{\text {eff }}$ column indicate the stars for which the $V$ band rather than the $J$ band was used for normalisation. The full machine-readable table is available online.

\begin{tabular}{lcccrr}
\hline Obj. Name & Spectral Type & $\begin{array}{r}T_{\text {eff }} \\
\mathrm{K}\end{array}$ & $\log g$ & {$[\mathrm{Fe} / \mathrm{H}]$} & $\begin{array}{r}E(B-V) \\
\mathrm{mag}\end{array}$ \\
\hline BD+00 2757 & F5 V: mF2gF5 & 6500 & 4.2 & -0.5 & 0.015 \\
CD-31 4428 & A2 Van & $8750 *$ & 4.2 & 0.0 & 0.05 \\
CD-58 3782 & A3 Van & 8700 & 4.2 & -0.2 & 0.03 \\
CD-60 1956 & A0.5 V & 9650 & 4.2 & 0.0 & 0.132 \\
CD-60 1986 & A2 Van & 9500 & 4.2 & 0.0 & 0.07 \\
CD-60 6017 & A8 IV-V & 7500 & 4.2 & 0.0 & 0.2 \\
CD-60 6021 & B7 IVn & $13000 *$ & 3.6 & 0.0 & 0.214 \\
CPD-20 1613 & A0.5 V kB9.5 & 9500 & 4.2 & -0.5 & 0.0 \\
CPD-58 3071 & A3 Va & $8500 *$ & 4.2 & 0.0 & 0.0 \\
CPD-58 3106 & A1.5 Vn & 9200 & 4.2 & 0.0 & 0.08 \\
HD 100237 & A1 IVs & 9500 & 3.6 & 0.0 & 0.0 \\
HD 100325 & A1 Va & 9500 & 4.2 & 0.0 & 0.172 \\
\hline
\end{tabular}

mined in Sec. 3.1. Bolometric corrections, BC, were computed via grid interpolation, taking the observed $T_{\text {eff }}, \log g$, and $[\mathrm{Fe} / \mathrm{H}]$ from SED fitting (Sec. 3.1) and uncertainties of $250 \mathrm{~K}, 0.5 \mathrm{dex}$ and $0.25 \mathrm{dex}$, respectively. These correspond to approximately $0.15 \mathrm{mag}, 0.02 \mathrm{mag}$ and $0.025 \mathrm{mag}$ of uncertainty in the $\mathrm{BC}$, which we combined in quadrature. We adopted a bolometric magnitude for the Sun, $M_{\text {bol, } \odot \text {, of } 4.74}$ (Mamajek et al. 2015). Distances were calculated using Gaia DR2 parallaxes (Gaia Collaboration et al. 2018), their uncertainties, and the length-scale model of Bailer-Jones et al. (2018). To determine luminosities with uncertainties, for each star we generated 10000 distance samples that we fed into a Monte Carlo process using equations 1 and 2, and took the median and standard deviation of the resulting distribution.

Using these luminosities together with the effective temperatures from SED fitting, we plot the $\lambda$ Boo stars in an HR diagram in Fig. 3 . The $\lambda$ Boo stars with infrared excesses are highlighted, some of which clearly lie near the terminal-age main sequence. This confirms earlier results (Paunzen et al. 2002, 2014; Murphy \& Paunzen 2017; Gray et al. 2017), that the $\lambda$ Boo stars have a range of main-sequence ages. There is no apparent preference towards the ZAMS, even among the $\lambda$ Boo stars with infrared excesses that are presumably attributable to discs.

\section{CONCLUSIONS}

The curation of a large and well defined sample of $\lambda$ Boo stars is important for understanding the accretion environments and particle transport processes affecting A type stars more broadly. We have classified 308 stars on the MK system and discovered or co-discovered 24 new $\lambda$ Boo stars, including two that require high-resolution spectroscopy for an abundance analysis to confirm their membership in the class. These represent a $17 \%$ increase in the number of known $\lambda$ Boo stars, adding to the 64 in the Murphy et al. (2015) catalogue and the 45 in Paper I, after accounting for overlap and revised spectral types. Our revision of 11 known $\lambda$ Boo stars revealed that one is a chemically normal rapid rotator. This one misclassified target suggests that abundance analyses would be valuable to confirm $\lambda$ Boo stars. 
Table 4. Breakdown of infrared excesses among the target selection groups described in Sec. 2.1. We give the number of $\lambda$ Boo stars, and their percentage of the total group numbers; the number of stars with IR excesses, and their percentage of the measurable population (i.e. group members where we could construct and evaluate SEDs for IR excesses); and the number of $\lambda$ Boo stars with IR excesses as a percentage of the number of $\lambda$ Boo stars in that group.

\begin{tabular}{|c|c|c|c|c|c|c|c|c|}
\hline \multirow[t]{2}{*}{ Group } & \multirow[t]{2}{*}{ Description } & \multirow{2}{*}{$\begin{array}{l}\text { Total } \\
\text { stars }\end{array}$} & \multicolumn{2}{|c|}{$\lambda \mathrm{Boo}$} & \multicolumn{2}{|c|}{ IR excess } & \multicolumn{2}{|c|}{$\lambda \mathrm{Boo}+\mathrm{IR}$} \\
\hline & & & Num. & $\%$ & Num. & $\%$ of group & Num. & $\%$ of $\lambda \mathrm{Boo}$ \\
\hline 0 & Known $\lambda$ Boo stars & 11 & 10 & 91 & 4 & 40 & 4 & 40 \\
\hline 1 & "A $[0-9]^{*} \mathrm{e} "$ & 20 & 4 & 20 & 17 & 85 & 4 & 100 \\
\hline 2 & "Em*/Ae*" and "A" & 18 & 1 & 6 & 16 & 89 & 1 & 100 \\
\hline 3 & Photometrically metal weak & 210 & 16 & 8 & 105 & 50 & 10 & 63 \\
\hline 4 & BHB stars & 7 & 2 & 29 & 4 & 57 & 1 & 50 \\
\hline $\mathrm{K} 2$ & K2 targets & 42 & 1 & 2 & 11 & 26 & 1 & 100 \\
\hline
\end{tabular}

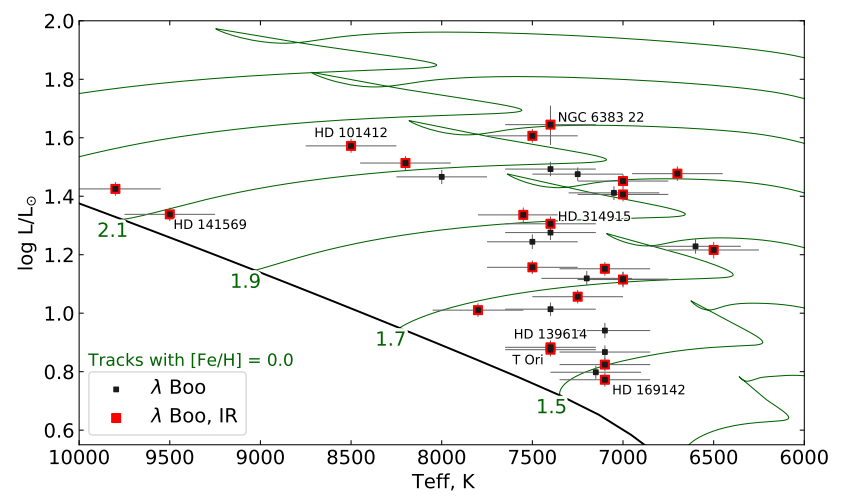

Figure 3. HR diagram of the $34 \lambda$ Boo stars. The 21 stars with infrared excesses are highlighted with red boxes and the seven stars with $>10 \sigma$ excesses are labelled (see also Fig. 1 and 2). Evolutionary tracks of solar metallicity from Murphy et al. (2019, green lines) are shown at intervals of $0.2 \mathrm{M}_{\odot}$.

The fraction of field A stars that are $\lambda$ Boo is known to be approximately $2 \%$, whereas stars identified photometrically as being metal-weak yielded a relatively high fraction of $\lambda$ Boo stars (8\%). We estimate that roughly half of all stars with Strömgren photometry and meeting our metal-weak criteria (Sec 2.1) have now been searched for $\lambda$ Boo stars, but with strong bias towards higher completeness in the southern hemisphere; the northern sky is comparatively underexplored, and will be the subject of future work, along with a refinement of those selection criteria to improve search efficiency. Our observations of 38 emission-line stars yielded 5 new $\lambda$ Boo stars (13\%). Emission-line stars are a relatively untapped source, since our search only used emission-line objects with known spectral types. Using further SIMBAD criteria searches, we find 1126 emission-line objects without spectral types but with the correct $B-V$ colours $(-0.05$ to $0.4)$ to be potential $\lambda$ Boo stars. These should be high priority targets for future searches for $\lambda$ Boo stars.

We collated fluxes in nine passbands to model the spectral energy distributions of all targets to look for infrared excesses. Unsurprisingly, infrared excesses were highly prevalent among the emission-line stars, including all those that are $\lambda$ Boo stars. We also calculated stellar luminosities to plot the $\lambda$ Boo stars on the HR diagram, confirming that not all $\lambda$ Boo stars are young: even those that have infrared excesses are found at a variety of main-sequence ages.

\section{ACKNOWLEDGEMENTS}

The authors thank the reviewer, Frédéric Royer, whose comments improved this manuscript. This work was supported by the Australian Research Council through the award DE180101104. This research has made use of the NASA/IPAC Infrared Science Archive, which is funded by the National Aeronautics and Space Administration and operated by the California Institute of Technology, It also made use of the SIMBAD database, operated at CDS, Strasbourg, France. We used Lightkurve, a Python package for Kepler and TESS data analysis (Lightkurve Collaboration et al. 2018), and SPECTRUM for creating synthetic spectra (Gray 1999).

\section{DATA AVAILABILITY}

Table A1, which appears in full in the appendix, is also available online. Tables 2 and 3, which contain stars with and without infrared excesses, respectively, are each shown for twelve rows in this paper and are available in full in machinereadable format online. The stellar spectra are available from the corresponding author upon reasonable request.

\section{REFERENCES}

Abt H. A., Morrell N. I., 1995, ApJS, 99, 135 Andrievsky S. M., et al., 2002, A\&A, 396, 641 Appenzeller I., Mundt R., 1989, A\&ARv, 1, 291

Bailer-Jones C. A. L., 2015, A\&A, 575, A35

Bailer-Jones C. A. L., Rybizki J., Fouesneau M., Mantelet G., Andrae R., 2018, AJ, 156, 58

Baschek B., Slettebak A., 1988, A\&A, 207, 112

Bessell M., Murphy S., 2012, PASP, 124, 140

Bodman E. H. L., Quillen A., 2016, ApJ, 819, L34

Boyajian T. S., et al., 2016, MNRAS, 457, 3988

Bruzzone J. S., et al., 2020, AJ, 159, 53

Carmona A., et al., 2017, A\&A, 598, A118

Carney M. T., et al., 2018, A\&A, 614, A106

Castelli F., Kurucz R. L., 2004, ArXiv Astrophysics e-prints,

Chen L., et al., 2019, ApJ, 887, L32

Cheng K.-P., et al., 2016, AJ, 151, 105 
Childress M. J., Vogt F. P. A., Nielsen J., Sharp R. G., 2014, Ap\&SS, 349, 617

Cowley C. R., Hubrig S., Castelli F., Wolff B., 2012, A\&A, 537, L6

Cugno G., et al., 2019, A\&A, 622, A156

Dopita M., Hart J., McGregor P., Oates P., Bloxham G., Jones D., 2007, Ap\&SS, 310, 255

Fedele D., et al., 2017, A\&A, 600, A72

Ferlet R., Hobbs L. M., Madjar A. V., 1987, A\&A, 185, 267

Fitzpatrick E. L., 1999, PASP, 111, 63

Folsom C. P., Bagnulo S., Wade G. A., Alecian E., Landstreet J. D., Marsden S. C., Waite I. A., 2012, MNRAS, 422, 2072

Freistetter F., Krivov A. V., Löhne T., 2007, A\&A, 466, 389

Gaia Collaboration et al., 2018, A\&A, 616, A1

Gratton R., et al., 2019, A\&A, 623, A140

Gray R. O., 1999, SPECTRUM: A stellar spectral synthesis program (ascl:9910.002)

Gray R. O., Corbally C. J., 1994, AJ, 107, 742

Gray R. O., Corbally C. J., 1998, AJ, 116, 2530

Gray R. O., Corbally C. J., 2002, AJ, 124, 989

Gray R. O., Corbally J. C., 2009, Stellar Spectral Classification. Princeton University Press

Gray R. O., Riggs Q. S., Koen C., Murphy S. J., Newsome I. M., Corbally C. J., Cheng K.-P., Neff J. E., 2017, AJ, 154, 31

Herbig G. H., 1960, ApJS, 4, 337

Hey D. R., et al., 2019, MNRAS, 488, 18

Hillenbrand L. A., Strom S. E., Vrba F. J., Keene J., 1992, ApJ, 397,613

Høg E., et al., 2000, A\&A, 355, L27

Holweger H., Sturenburg S., 1993, in Dworetsky M. M., Castelli F., Faraggiana R., eds, Astronomical Society of the Pacific Conference Series Vol. 44, IAU Colloq. 138: Peculiar versus Normal Phenomena in A-type and Related Stars. p. 356

Howell S. B., et al., 2014, PASP, 126, 398

Iliev I. K., Barzova I. S., 1995, A\&A, 302, 735

Jermyn A. S., Kama M., 2018, MNRAS, 476, 4418

Joy A. H., 1945, ApJ, 102, 168

Jura M., 2015, AJ, 150, 166

Kama M., Folsom C. P., Pinilla P., 2015, A\&A, 582, L10

Kamp I., Paunzen E., 2002, MNRAS, 335, L45

Kamp I., Iliev I. K., Paunzen E., Pintado O. I., Solano E., Barzova I. S., 2001, A\&A, 375, 899

Karmann C., Beust H., Klinger J., 2001, A\&A, 372, 616

Karmann C., Beust H., Klinger J., 2003, A\&A, 409, 347

King J. R., 1994, MNRAS, 269, 209

King J. R., Patten B. M., 1992, MNRAS, 256, 571

Lagrange A.-M., et al., 2010, Science, 329, 57

Laws A. S. E., et al., 2020, ApJ, 888, 7

Lellouch E., et al., 1997, Planet. Space Sci., 45, 1203

Lightkurve Collaboration et al., 2018, Lightkurve: Kepler and TESS time series analysis in Python, Astrophysics Source Code Library (ascl:1812.013)

Ligi R., et al., 2018, MNRAS, 473, 1774

MacConnell D. J., Frye R. L., Bidelman W. P., Bond H. E., 1971, PASP, 83, 98

Macías E., et al., 2019, ApJ, 881, 159

Mamajek E. E., et al., 2015, preprint, (arXiv:1510.06262)

Matter A., et al., 2016, A\&A, 586, A11

Mawet D., et al., 2017, AJ, 153, 44

Mendigutía I., Oudmaijer R. D., Mourard D., Muzerolle J., 2017, MNRAS, 464, 1984

Miley J. M., Panić O., Wyatt M., Kennedy G. M., 2018, A\&A, $615, \mathrm{~L} 10$

Murphy S. J., Paunzen E., 2017, MNRAS, 466, 546

Murphy S. J., et al., 2013, MNRAS, 432, 2284

Murphy S. J., et al., 2015, Publ. Astron. Soc. Australia, 32, 36

Murphy S. J., Hey D., Van Reeth T., Bedding T. R., 2019, MNRAS, 485,2380
Murphy S. J., Paunzen E., Bedding T. R., Walczak P., Huber D., 2020, MNRAS, 495, 1888

Nesterov V. V., Kuzmin A. V., Ashimbaeva N. T., Volchkov A. A., Röser S., Bastian U., 1995, A\&AS, 110, 367

Paunzen E., 2001, A\&A, 373, 633

Paunzen E., Gray R. O., 1997, A\&AS, 126, 407

Paunzen E., Duffee B., Heiter U., Kuschnig R., Weiss W. W., 2001, A\&A, 373, 625

Paunzen E., Iliev I. K., Kamp I., Barzova I. S., 2002, MNRAS, 336,1030

Paunzen E., Iliev I. K., Fossati L., Heiter U., Weiss W. W., 2014, A\&A, 567, A67

Schöller M., et al., 2016, A\&A, 592, A50

Slettebak A., 1952, ApJ, 115, 575

Slettebak A., 1954, ApJ, 119, 146

Smith M. A., Thompson R. W., Gray R. O., Corbally C., Kamp I., 2011, arXiv e-prints, p. arXiv: 1112.3617

Snellen I. A. G., Brown A. G. A., 2018, Nature Astronomy, 2, 883

Soummer R., Brendan Hagan J., Pueyo L., Thormann A., Rajan A., Marois C., 2011, ApJ, 741, 55

Thébault P., Beust H., 2001, A\&A, 376, 621

Toci C., Lodato G., Fedele D., Testi L., Pinte C., 2020, ApJ, 888, L4

Turcotte S., Charbonneau P., 1993, ApJ, 413, 376

Venn K. A., Lambert D. L., 1990, ApJ, 363, 234

Waters L. B. F. M., Trams N. R., Waelkens C., 1992, A\&A, 262, L37

Wenger M., et al., 2000, A\&AS, 143, 9

White J. A., Boley A. C., MacGregor M. A., Hughes A. M., Wilner D. J., 2018, MNRAS, 474, 4500

Xue M., Jiang B. W., Gao J., Liu J., Wang S., Li A., 2016, ApJS, 224,23

Zieba S., Zwintz K., Kenworthy M. A., Kennedy G. M., 2019, A\&A, 625, L13

Zuckerman B., Song I., 2012, ApJ, 758, 77

This paper has been typeset from a $\mathrm{T}_{\mathrm{E}} \mathrm{X} / \mathrm{L} \mathrm{LT}_{\mathrm{E}} \mathrm{X}$ file prepared by the author.

\section{APPENDIX A: SPECTRAL CLASSES FOR THE PROGRAM STARS}


Table A1. Spectral classes for the program stars. We give the target group of each star (Sec. 2.1). Comments on the spectra are recorded as endnotes. Infrared excesses are denoted with ' 1 ' in the final column.

\begin{tabular}{|c|c|c|c|c|c|}
\hline Obj Name & $\mathrm{V}$ mag & Group & Class & Note & IR \\
\hline HD 28490 & 9.53 & 3 & F0 V(n) kA5mA5 ( $\lambda$ Boo $)$ & 2 & \\
\hline HD 29650 & 9.66 & 3 & A3 IV-Vs & & 1 \\
\hline HD 30335 & 9.67 & 2 & A4 IV Sr & & \\
\hline HD 32725 & 9.52 & 3 & F3 V Sr & 3 & 1 \\
\hline HD 33901 & 9.52 & 3 & A7 III & & \\
\hline HD 35343 & 10.25 & 1 & $\mathrm{Be} 3$ & 4 & 1 \\
\hline HD 35793 & 9.77 & 3 & A2 Vs & & 1 \\
\hline HD 36121 & 8.98 & 1 & kA6hA8mA8 III Sr & 5 & \\
\hline HD 36866 & 9.52 & 3 & A3 Vas & & 1 \\
\hline HD 36899 & 9.8 & 3 & $\mathrm{~A} 1 \mathrm{~V}$ & & \\
\hline HD 36955 & 9.58 & 3 & $\mathrm{~A} 7 \mathrm{Vp} \mathrm{SrEu}$ & & \\
\hline HD 37091 & 9.82 & 3 & $\mathrm{~A} 2.5 \mathrm{~V}$ & & 1 \\
\hline HD 37258 & 9.61 & 3 & A3 V shell & 6 & 1 \\
\hline HD 37357 & 8.85 & 1 & A3 Van kA1 & & 1 \\
\hline HD 37412 & 9.76 & 3 & $\mathrm{~A} 2.5 \mathrm{Vs}$ & & 1 \\
\hline HD 37455 & 9.6 & 3 & $\mathrm{~A} 3 \mathrm{Vb}$ & & 1 \\
\hline HD 37469 & 9.58 & 3 & B9 $\mathrm{Vp} \mathrm{Si}-\mathrm{Sr}$ & & 1 \\
\hline HD 40632 & 9.15 & 1 & B9 IV shell & 7 & 1 \\
\hline HD 44351 & 8.25 & 1 & F5 V composite & 8 & 1 \\
\hline HD 46390 & 10.08 & 2 & B7 IV-Ve & 9 & 1 \\
\hline HD 50937 & 9.61 & 3 & A2 IVn & & \\
\hline HD 51480 & 6.93 & 2 & B3/5 Ibe & 10 & 1 \\
\hline HD 55637 & 9.65 & 2 & B6 IV & 11 & 1 \\
\hline HD 59000 & 9.57 & 3 & $\mathrm{~A} 8 \mathrm{IV} / \mathrm{V}$ & & \\
\hline HD 62752 & 8.11 & 3 & B9 Vp SiEuSr & 12 & \\
\hline HD 63524 & 8.8 & 2 & B6.5 Vn & & \\
\hline HD 63562 & 9.69 & 3 & $\mathrm{~A} 1 \mathrm{~V}$ & & 1 \\
\hline HD 66318 & 9.56 & 3 & A2: IV:p SiSrCr & 13 & \\
\hline HD 67658 & 9.76 & 3 & A4 IV & & \\
\hline HD 68695 & 9.87 & 1 & A3 VbekA0mA0.5 & 14 & 1 \\
\hline HD 75185 & 9.82 & 3 & A2 IV-n. & & \\
\hline HD 79066 & 6.34 & 1 & $\mathrm{~F} 1 \mathrm{Vn}$ & 15 & \\
\hline HD 80692 & 9.69 & 3 & FoV + Ae composite & 16 & \\
\hline HD 83041 & 8.79 & 4 & $\mathrm{~F} 1.5 \mathrm{VkA} 3 \mathrm{~mA} 3 \lambda$ Boo & & \\
\hline HD 83798 & 9.58 & 3 & A5 IVnn & & \\
\hline HD 85337 & 9.61 & 3 & hA9 Vn kA6mA6 & 17 & \\
\hline HD 87271 & 7.13 & 0 & $\mathrm{~A} 8 \mathrm{VkA} 0 \mathrm{~mA} 0.5 \lambda$ Boo & & 1 \\
\hline HD 87593 & 9.62 & 3 & A2 Vas & & \\
\hline HD 88554 & 9.32 & 3 & F3 VkA6mA8 $(\lambda \mathrm{Boo})$ & & 1 \\
\hline HD 88976 & 6.54 & 3 & A2 IV-V & & 1 \\
\hline HD 89234 & 9.79 & 3 & A $0.5 \mathrm{IV}$ & & 1 \\
\hline HD 91839 & 8.39 & 3 & A3 Vas (met wk A2) & 18 & 1 \\
\hline HD 92251 & 9.81 & 3 & A 0.5 Vas & & \\
\hline HD 93264 & 9.54 & 3 & $\mathrm{kA} 1.5 \mathrm{hA} 2 \mathrm{~mA} 3 \mathrm{IV}-\mathrm{V}$ & 19 & \\
\hline HD 93746 & 9.52 & 3 & F $3 \mathrm{~V}$ & & \\
\hline HD 93925 & 9.24 & 3 & A0 II-IIIp Eu & & 1 \\
\hline HD 94326 & 7.76 & 3 & A 6 III kA5 & & 1 \\
\hline HD 95883 & 7.33 & 3 & A1 Van & 20 & 1 \\
\hline HD 96040 & 9.97 & 3 & B9 IIIp Si & & 1 \\
\hline HD 96089 & 9.78 & 3 & A1 Van & & 1 \\
\hline HD 96091 & 9.57 & 3 & A 0.5 Van & & \\
\hline HD 96157 & 9.82 & 3 & A2 Van & & \\
\hline HD 96192 & 9.66 & 3 & A3 Van kA1 & & 1 \\
\hline HD 96304 & 9.54 & 3 & A0.5 Van & & 1 \\
\hline HD 96341 & 9.53 & 3 & A $0.5 \mathrm{Van}$ & & 1 \\
\hline HD 96386 & 9.83 & 3 & A2 IV-V & & 1 \\
\hline HD 96430 & 8.49 & 2 & B6 IV/Ve & 21 & 1 \\
\hline HD 96493 & 8.5 & 3 & A0.5 III shell & & 1 \\
\hline
\end{tabular}

\footnotetext{
2 A mild $\lambda$ Boo star. Ba dwarf.

${ }_{5}^{4}$ Strong emission in $\mathrm{H}$ lines and Fe II lines

5 Mild Am peculiarity.

6 Shell core in $\mathrm{H} \beta$ and Fe II 4233.
}

3 Especially clear enhancement of Sr II 4215. Might be an early

\footnotetext{
${ }^{7}$ Strong metallic-line spectrum, similar to F0 III. The Fe II 4233 line is strong and the hydrogen line cores are deep.

8 The $\mathrm{K}$ line is broad and shallow, while metal lines are of mixed strengths.

9 Emission reversal in $\mathrm{H} \beta . \mathrm{H} \gamma$ and $\mathrm{H} \delta$ partially filled with emission.

10 P Cygni profile

11 Classified in Simbad as emission line Ap Si. No sign of emission or increased abundance of $\mathrm{Si}$.

12 Very peculiar.

13 Very peculiar. Temperature type very uncertain. H lines do
} not fit well at any spectral type.

${ }^{14}$ Emission in the core of $\mathrm{H} \boldsymbol{\beta}$. Mg II 4481 is normal. Not a $\lambda$ Boo star.

15 Rapid rotation gives the impression of metal weakness unless comparing against high $v \sin i$ standards.

${ }^{16} \mathrm{H} \beta$ has broad wings and a deep narrow core, suggesting shell, emission, or composite. K-line about A1. $\mathrm{H} \gamma$ is F0 V. Note that Simbad has this as an eclipsing binary.

17 More rapidly rotating than the Vn standards. Metal weak even after considering rotation.

18 Not a $\lambda$ Boo star.

19 A mild Am star.

20 Shallow H cores.

21 Emission in core of $\mathrm{H} \beta$, possibly causing other $\mathrm{H}$ lines to be shallower.

${ }^{22}$ Not an Am star since the $\mathrm{K}$ line is strong, too.

23 A mild $\lambda$ Boo star, with fluted $\mathrm{H} \gamma$ lines

24 A $\rho$ Pup star.

25 Mild Am, with anomalous luminosity effect.

26 Marginal Am star. Anomalous luminosity effect evident.

27 Metal weak overall, but less so in K line. Weakness of Ca I 4226 and the difference between the $\mathrm{K}$ and $\mathrm{m}$ types suggest this is not a $\lambda$ Boo star, despite weak Mg II 4481 line. May be composite.

28 Slight emission notch in $\mathrm{H} \beta$. A mild $\lambda$ Boo star.

29 Not a $\lambda$ Boo star - Mg II 4481 is not additionally weak. Fe I 4046 is peculiarly strong in absorption.

${ }^{30} \mathrm{H}$ lines fit best at A1 V, and are too narrow for A2. A2 IV is not as good a fit as A1 V. Metal lines and $\mathrm{K}$ line are strong for A1, being $\sim \mathrm{A} 2$.

31 Not a $\lambda$ Boo star, just marginally metal weak.

32 Very peculiar spectrum. Very weak $\mathrm{H}$ lines, which can be approximately fitted by a B7 supergiant, or a mid-late F supergiant. In the case of the latter, the star is profoundly metal weak. Most likely a pop II star, possibly a high-latitude F supergiant, although the spectrum is peculiar even for that class.

33 Very chemically peculiar star. H lines much deeper than A0 III, but wings agree.

${ }^{34}$ Luminosity criteria (e.g. $\lambda \lambda 4172-8$ ) do not agree with the hydrogen line type of A3 II, but otherwise a good match.

35 Not a $\lambda$ Boo star, maybe pop II or composite A + F.

${ }^{36}$ Definite $\lambda$ Boo star. Mg II 4481 is extremely weak.

37 Highly peculiar star. $\mathrm{H}$ lines, especially. $\mathrm{H} \beta$ is deeper than the standard, may be shell absorption.

38 Slightly weak K line (A2).

39 Excellent match to standard, HR 2324.

40 Extreme $\lambda$ Boo star.

${ }^{41} \mathrm{H}$ lines are truly halfway between A0 Van and A1 Van. Metal lines are consistent with this. 
Table A1. continued. Spectral classes for the program stars.

\begin{tabular}{|c|c|c|c|c|c|}
\hline Obj Name & $\mathrm{V}$ mag & Group & Class & Note & IR \\
\hline HD 96667 & 9.58 & 3 & A1 Van & & \\
\hline HD 96773 & 9.69 & 3 & A1 Van & & 1 \\
\hline HD 97230 & 8.62 & $\mathrm{~K} 2$ & A7 IV (met str F2) & 22 & \\
\hline HD 97340 & 8.15 & $\mathrm{~K} 2$ & A9 V mA6 & & \\
\hline HD 97373 & 8.67 & K2 & A4IVn & & \\
\hline HD 97528 & 7.31 & 3 & A2 IIIe shell & & 1 \\
\hline HD 97678 & 8.67 & K2 & $\mathrm{F} 2 \mathrm{Vs}$ & & \\
\hline HD 97859 & 9.35 & $\mathrm{~K} 2$ & B8 IVp Si & & \\
\hline HD 97891 & 8.33 & $\mathrm{~K} 2$ & $\mathrm{~F} 5.5 \mathrm{~V}$ & & \\
\hline HD 97916 & 9.2 & $\mathrm{~K} 2$ & $\mathrm{~F} 5.5 \mathrm{~V} \mathrm{gF} 2.5 \mathrm{kF} 2: \mathrm{mA} 6$ & & 1 \\
\hline HD 97991 & 7.41 & $\mathrm{~K} 2$ & $\mathrm{~B} 1 \mathrm{~V}$ & & 1 \\
\hline HD 98069 & 8.16 & $\mathrm{~K} 2$ & A9 VkA2mA2 $(\lambda$ Boo $)$ & 23 & 1 \\
\hline HD 98563 & 8.27 & $\mathrm{~K} 2$ & $\mathrm{~F} 7 \mathrm{~V}$ & & \\
\hline HD 98575 & 9.12 & $\mathrm{~K} 2$ & kA5hA9mF3 III & 24 & \\
\hline HD 98632 & 7.57 & $\mathrm{~K} 2$ & F4 Vs mF1 & & 1 \\
\hline HD 98645 & 8.8 & K2 & F1 Vs & & \\
\hline HD 98686 & 7.65 & $\mathrm{~K} 2$ & A $8 \mathrm{Vnn}$ & & \\
\hline HD 98711 & 8.07 & $\mathrm{~K} 2$ & F6 IV-V & & \\
\hline HD 98914 & 8.08 & $\mathrm{~K} 2$ & F5.5 V & & \\
\hline HD 99210 & 6.74 & $\mathrm{~K} 2$ & kA8hA9mF2 III: & 25 & 1 \\
\hline HD 99304 & 8.58 & $\mathrm{~K} 2$ & F5 IV & & \\
\hline HD 99776 & 9.18 & $\mathrm{~K} 2$ & A2.5 Vas & & \\
\hline HD 100237 & 7.34 & K2 & A1 IVs & & \\
\hline HD 100325 & 9.28 & 3 & $\mathrm{~A} 1 \mathrm{Va}$ & & \\
\hline HD 100380 & 6.78 & 3 & A4 IVs & & 1 \\
\hline HD 100415 & 9.06 & $\mathrm{~K} 2$ & kA6hA8mF1 (IV-III) & 26 & \\
\hline HD 100417 & 8.03 & $\mathrm{~K} 2$ & A1 Vas & & \\
\hline HD 100453 & 7.79 & 1 & F1 Vn & & 1 \\
\hline HD 100630 & 7.88 & $\mathrm{~K} 2$ & $\mathrm{~A} 1.5 \mathrm{Va}$ & & \\
\hline HD 100762 & 9.32 & K2 & $\mathrm{F} 4 \mathrm{Vs}$ & & \\
\hline HD 100995 & 8.09 & $\mathrm{~K} 2$ & $\mathrm{~F} 4.5 \mathrm{~V}$ & & \\
\hline HD 101196 & 8.5 & K2 & $\mathrm{F} 4 \mathrm{Vs}$ & & \\
\hline HD 101268 & 9.55 & 3 & F1 VskA8mA6 & 27 & \\
\hline HD 101412 & 9.29 & 3 & $\mathrm{~A} 3 \mathrm{~V}(\mathrm{e}) \mathrm{kA} 0.5 \mathrm{~mA} 0.5(\lambda \mathrm{Boo})$ & 28 & 1 \\
\hline HD 101784 & 7.54 & $\mathrm{~K} 2$ & A0 Vas & & \\
\hline HD 101846 & 7.87 & $\mathrm{~K} 2$ & $\mathrm{~A} 4 \mathrm{Vs}$ & & 1 \\
\hline HD 101969 & 7.54 & $\mathrm{~K} 2$ & $\mathrm{~F} 4 \mathrm{~V}$ & & 1 \\
\hline HD 102059 & 7.76 & $\mathrm{~K} 2$ & $\mathrm{~F} 4 \mathrm{Vs}$ & & 1 \\
\hline HD 102083 & 8.58 & $\mathrm{~K} 2$ & F0 V mA7 & & \\
\hline HD 102284 & 8.54 & $\mathrm{~K} 2$ & F3 IVs & & 1 \\
\hline HD 102331 & 7.57 & $\mathrm{~K} 2$ & $\mathrm{~F} 4 \mathrm{Vs}$ & & \\
\hline HD 102332 & 8.51 & $\mathrm{~K} 2$ & F4 IVs & & 1 \\
\hline HD 102431 & 8.95 & $\mathrm{~K} 2$ & F $5.5 \mathrm{~V}$ & & \\
\hline HD 102519 & 8.66 & 3 & A1 IVn & & 1 \\
\hline HD 102541 & 7.94 & 3 & hA9 V kA5mA6 & 29 & \\
\hline HD 102731 & 8.49 & $\mathrm{~K} 2$ & A6 IVs & & \\
\hline HD 103547 & 9.38 & $\mathrm{~K} 2$ & $\mathrm{~F} 1 \mathrm{Vs} \mathrm{mF} 2.5$ & & 1 \\
\hline HD 103631 & 8.53 & $\mathrm{~K} 2$ & F8 IV & & \\
\hline HD 103695 & 8.52 & $\mathrm{~K} 2$ & $\mathrm{~A} 6 \mathrm{~V}$ & & \\
\hline HD 104367 & 7.78 & $\mathrm{~K} 2$ & F6 IV & & \\
\hline HD 104446 & 9.05 & 3 & A1 IVs & & 1 \\
\hline HD 104624 & 9.13 & $\mathrm{~K} 2$ & $\mathrm{~A} 4 \mathrm{~V}$ & & \\
\hline HD 104650 & 9.78 & 3 & A1 Vas mA2 & 30 & 1 \\
\hline HD 104697 & 9.46 & 3 & $\mathrm{~A} 1 \mathrm{Va}$ & & 1 \\
\hline HD 105015 & 8.64 & 3 & A1 Vas & & \\
\hline HD 105194 & 9.32 & 3 & hA0.5 Van kB9.5mA0 & 31 & \\
\hline HD 105209 & 8.67 & 3 & A2 IVn & & 1 \\
\hline HD 105232 & 8.66 & 3 & $\mathrm{~A} 2 \mathrm{Vs}$ & & \\
\hline HD 105649 & 9.83 & 3 & A2 IV-V & & 1 \\
\hline HD 106373 & 8.9 & 4 & F5: Ia: kA3mA3 & 32 & 1 \\
\hline HD 106961 & 8.93 & 3 & A0 Vann & & \\
\hline HD 107049 & 9.36 & 3 & $\mathrm{~A} 0 \mathrm{IV}+$ & & 1 \\
\hline
\end{tabular}

42 Composite spectrum.

43 Late $\lambda$ Boo candidate. Needs abundance analysis to decide.

$44 \mathrm{H}$ lines are not consistent, F0/3.

45 Difference in $\mathrm{K}$ and $\mathrm{M}$ type argues against a $\lambda$ Boo classification.

46 Hydrogen line is at A9, when comparing to the A9 Vn standard.

47 Slight emission notch in $\mathrm{H} \beta$. Mild $\lambda$ Boo star - slight additional weakness in the Mg II 4481 line.
Table A1. continued. Spectral classes for the program stars.

\begin{tabular}{|c|c|c|c|c|c|}
\hline Obj Name & $\mathrm{V}$ mag & Group & Class & Note & IR \\
\hline HD 107096 & 9.37 & 3 & A0: III:p Eu & 33 & 1 \\
\hline HD 107127 & 9.57 & 3 & A1 IV & & \\
\hline HD 107233 & 7.36 & 0 & F0 V kA3mA2 $\lambda$ Boo & & \\
\hline HD 107369 & 9.6 & 4 & A3 IIp & 34 & 1 \\
\hline HD 107483 & 9.3 & 3 & A1 IV-III & & \\
\hline HD 107878 & 9.71 & 3 & A9 V mA2 & 35 & \\
\hline HD 108417 & 8.98 & 3 & $\mathrm{~A} 2 \mathrm{~V}$ & & 1 \\
\hline HD 108889 & 8.86 & 3 & A1 IVs & & 1 \\
\hline HD 108925 & 6.45 & 3 & A3IVn & & \\
\hline HD 109065 & 8.16 & 3 & A1 IVn & & \\
\hline HD 109183 & 9.1 & 3 & $\mathrm{~A} 1 \mathrm{Va}+\mathrm{s}$ & & \\
\hline HD 109435 & 8.99 & 3 & A7 IV & & 1 \\
\hline HD 109443 & 9.25 & 3 & $\mathrm{~F} 3 \mathrm{~V} \mathrm{kF} 1 \mathrm{mF} 1$ & & \\
\hline HD 109517 & 8.77 & 3 & kA0hA0mA1 IV-V & & 1 \\
\hline HD 109738 & 8.3 & 0 & hA9 Vn kA0mA0 $\lambda$ Boo & 36 & \\
\hline HD 109791 & 9.75 & 3 & A0 II-IIIp SrSiEu shell? & 37 & \\
\hline HD 109800 & 8.84 & 3 & $\mathrm{~A} 1 \mathrm{IV}+\mathrm{s}$ & & 1 \\
\hline HD 109808 & 7.13 & 3 & A3 V & 38 & \\
\hline HD 109886 & 8.61 & 3 & A1 Van & 39 & \\
\hline HD 110640 & 9.0 & 3 & A2 Vas & & \\
\hline HD 111105 & 7.25 & 3 & A3IVn & & 1 \\
\hline HD 111164 & 6.09 & 0 & $\mathrm{~A} 4 \mathrm{~V}(\mathrm{n})$ & & \\
\hline HD 111209 & 9.62 & 3 & $\mathrm{~A} 4 \mathrm{Vn}$ & & 1 \\
\hline HD 111438 & 9.18 & 3 & A 1.5 Vas & & \\
\hline HD 111439 & 8.87 & 3 & A3 IV-Vs & & \\
\hline HD 111786 & 6.14 & 0 & F0 VskA1mA $1 \lambda$ Boo & 40 & 1 \\
\hline HD 112938 & 8.16 & 3 & A2 IV-V & & 1 \\
\hline HD 113199 & 8.81 & 3 & $\mathrm{~A} 2 \mathrm{~V}$ & & 1 \\
\hline HD 113660 & 9.32 & 3 & A6 IVs & & 1 \\
\hline HD 113807 & 7.56 & 3 & A2 Vas & & \\
\hline HD 114477 & 8.4 & 3 & $\mathrm{~A} 1.5 \mathrm{IVs}$ & & \\
\hline HD 114738 & 7.81 & 3 & A1 IV-V & & \\
\hline HD 114836 & 8.74 & 3 & A0.5 Van & 41 & \\
\hline HD 115843 & 9.34 & 3 & A1 IVs & & \\
\hline HD 116137 & 9.09 & 3 & B9.5 Van & & \\
\hline HD 119561 & 9.79 & 3 & A1 Van & & \\
\hline HD 119896 & 8.22 & 3 & F5 Vs kA5mA $5 \lambda$ Boo & & 1 \\
\hline HD 120122 & 9.11 & 3 & F1 VskA6mA6 ( $\lambda$ Boo $)$ & & \\
\hline HD 120873 & 9.36 & 3 & A1 Van & & \\
\hline HD 121875 & 9.26 & 3 & A2 IV-Vn & & \\
\hline HD 122264 & 9.59 & 3 & A0 IIIp EuSr & & \\
\hline HD 122757 & 8.8 & 3 & $\mathrm{~A} 3 \mathrm{Va}+\mathrm{s}$ & & \\
\hline HD 123960 & 9.75 & 3 & B9 IIIp Si & & 1 \\
\hline HD 124228 & 7.86 & 3 & $\mathrm{~A} 3 \mathrm{IV}+\mathrm{s}$ & & \\
\hline HD 124878 & 9.54 & 3 & $\mathrm{~B} 8 \mathrm{~V} \mathrm{He}-\mathrm{wk}+\mathrm{A}$ & 42 & \\
\hline HD 126164 & 9.41 & 3 & A0 Vbn & & \\
\hline HD 126627 & 9.0 & 3 & $\mathrm{~F} 1 \mathrm{VkA} 5 \mathrm{~mA} 5(\lambda \mathrm{Boo})$ & & 1 \\
\hline HD 127659 & 9.31 & 3 & $\mathrm{~F} 2 \mathrm{VkA} 3 \mathrm{~mA} 4 \lambda \mathrm{Boo}$ & & 1 \\
\hline HD 128336 & 9.08 & 3 & $\mathrm{~F} 4 \mathrm{VkA} 2 \mathrm{~mA} 2 \lambda \mathrm{Boo} ?$ & 43 & \\
\hline HD 129389 & 9.68 & 3 & A0 Van & & 1 \\
\hline HD 130156 & 9.35 & 4 & kA6hF1mF3 (II) & 44 & \\
\hline HD 133800 & 6.4 & 3 & $\mathrm{~A} 6 \mathrm{VkA} 0.5 \mathrm{~mA} 0.5 \lambda \mathrm{Boo}$ & & 1 \\
\hline HD 134685 & 7.67 & 3 & $\mathrm{~A} 1 \mathrm{~V}$ & & 1 \\
\hline HD 135284 & 9.23 & 3 & $\mathrm{~A} 3 \mathrm{IV}+\mathrm{s}$ & & \\
\hline HD 136463 & 9.54 & 3 & $\mathrm{~F} 1 \mathrm{VkF} 1 \mathrm{~mA} 7$ & 45 & \\
\hline HD 137128 & 7.1 & 3 & A3 IV-V & & \\
\hline HD 138274 & 8.8 & 3 & A1 IVs & & \\
\hline HD 138753 & 8.54 & 3 & A0.5 Van & & \\
\hline HD 138921 & 9.72 & 3 & A9 Vn kA4mA4 $\lambda$ Boo & 46 & \\
\hline HD 139612 & 9.27 & 3 & A0 IVs & & \\
\hline HD 139614 & 8.24 & 1 & A9 Vs $(\mathrm{e}) \mathrm{kA} 5 \mathrm{~mA} 7$ ( $\lambda$ Boo $)$ & 47 & 1 \\
\hline HD 139787 & 9.77 & 3 & $\mathrm{~A} 4 \mathrm{~V}$ & & \\
\hline
\end{tabular}

48 Great match to the A5IV standard, $\beta$ Tri, except for the Ca I 4226 line, which is much stronger than in the standard. 49 Mild Am.

50 The Mg II 4481 line is weak, but this is not a $\lambda$ Boo star, since the $\mathrm{K}$ line is normal.

51 Slightly shallow H cores. 
Table A1. continued. Spectral classes for the program stars.

\begin{tabular}{|c|c|c|c|c|c|}
\hline Obj Name & $\mathrm{V}$ mag & Group & Class & Note & IR \\
\hline HD 140734 & 9.55 & 3 & A5 IV & 48 & \\
\hline HD 141063 & 6.98 & 3 & $\mathrm{kA} 2 \mathrm{hA} 3 \mathrm{~mA} 5 \mathrm{Va}+$ & 49 & \\
\hline HD 141403 & 9.03 & 3 & A1 Vbs & & 1 \\
\hline HD 141442 & 8.74 & 3 & $\mathrm{~A} 1 \mathrm{Va}$ & & \\
\hline HD 141444 & 8.94 & 3 & $\mathrm{~A} 0 \mathrm{Va}$ & 50 & \\
\hline HD 141569 & 7.12 & 1 & A1 Vn kB9mB9 $\lambda$ Boo & & 1 \\
\hline HD 141576 & 9.04 & 3 & A 0.5 Vas & & \\
\hline HD 141905 & 8.3 & 3 & $\mathrm{~A} 2 \mathrm{Va}+\mathrm{n}$ & & \\
\hline HD 142404 & 9.14 & 3 & A1.5 Vas & 51 & \\
\hline HD 142524 & 9.59 & 3 & A1 Van & & \\
\hline HD 142666 & 8.82 & 1 & F0 V shell & 52 & 1 \\
\hline HD 142703 & 6.12 & 0 & F1 Vs kA1.5mA1.5 $\lambda$ Boo & & \\
\hline HD 142705 & 7.74 & 3 & A1 Vann & & 1 \\
\hline HD 142931 & 9.79 & 3 & A1.5 Vas & & \\
\hline HD 142994 & 7.17 & 0 & hF2 VkA5mA5 ( $\lambda$ Boo) & & 1 \\
\hline HD 143511 & 8.31 & 3 & A1 Vas & 53 & \\
\hline HD 143567 & 7.19 & 3 & B9 Van & 54 & \\
\hline HD 143600 & 7.33 & 3 & B9 Vn & 55 & 1 \\
\hline HD 143715 & 7.14 & 3 & A1.5 IVs & 56 & 1 \\
\hline HD 143747 & 8.4 & 3 & A1 IVn & & \\
\hline HD 143822 & 9.39 & 3 & A1 Vbn & & \\
\hline HD 143956 & 7.77 & 3 & B9 Van & & 1 \\
\hline HD 144254 & 7.78 & 3 & A1 Van kA0.5 & & 1 \\
\hline HD 144273 & 7.54 & 3 & B9 Vn & & 1 \\
\hline HD 144569 & 7.9 & 3 & A1 Vas mA0.5 & 57 & 1 \\
\hline HD 144586 & 7.81 & 3 & A1 IV-V kB9 & 58 & 1 \\
\hline HD 144668 & 7.05 & 1 & A9 V shell & 59 & 1 \\
\hline HD 144925 & 7.78 & 3 & A0 Vn & 60 & 1 \\
\hline HD 144981 & 8.04 & 3 & $\mathrm{~A} 0.5 \mathrm{Vn}$ & & 1 \\
\hline HD 145188 & 7.06 & 3 & $\mathrm{~B} 9.5 \mathrm{Vb}$ & & 1 \\
\hline HD 145631 & 7.6 & 3 & B9 Vann & & 1 \\
\hline HD 146706 & 7.55 & 3 & B9 Van & & 1 \\
\hline HD 147010 & 7.4 & 3 & B8: Vp SrTi SiEu & & 1 \\
\hline HD 147046 & 7.8 & 3 & A2 IVn & 61 & 1 \\
\hline HD 148036 & 9.62 & 3 & $\mathrm{~F} 0.5 \mathrm{Vn} \mathrm{kA} 6 \mathrm{~mA} 6$ & 62 & \\
\hline HD 148534 & 9.02 & 3 & A2 Vas & & \\
\hline HD 148563 & 8.72 & 3 & $\mathrm{~A} 2 \mathrm{Va}$ & & 1 \\
\hline HD 148638 & 7.9 & 3 & A2 IV-Vn & & 1 \\
\hline HD 149130 & 8.48 & 3 & $\mathrm{~F} 1 \mathrm{Vp} \mathrm{Sr}$ & 63 & 1 \\
\hline HD 149151 & 8.12 & 3 & A0 IV-V SrSi & & \\
\hline HD 150035 & 8.71 & 3 & A2 IVp SrCrEu & & 1 \\
\hline HD 150193 & 8.79 & 1 & A3 Va(e) & 64 & 1 \\
\hline HD 151873 & 9.1 & 2 & B9 III shell & 65 & 1 \\
\hline HD 153747 & 7.42 & 0 & $\mathrm{~A} 6 \mathrm{VnkA0mA0} \lambda \mathrm{Boo}$ & 66 & \\
\hline HD 153948 & 9.54 & 3 & B9 IVp SiSrCrEu & & 1 \\
\hline HD 154153 & 6.18 & 3 & $\mathrm{~F} 1.5 \mathrm{Vs} \mathrm{kA} 4 \mathrm{~mA} 4 \lambda \mathrm{Boo}$ & & 1 \\
\hline HD 154751 & 8.96 & 3 & A3 IV & & \\
\hline HD 154951 & 8.78 & 3 & F2 VkA6mA6 $((\lambda$ Boo $))$ & 67 & \\
\hline HD 155127 & 8.38 & 3 & kA0hA5mF0 II & & \\
\hline HD 155397 & 9.53 & 3 & $\mathrm{~F} 2 \mathrm{Vs}$ & & 1 \\
\hline HD 156300 & 8.65 & 3 & A1: IIIp EuCr(Sr) & & \\
\hline HD 156974 & 9.39 & 3 & A0.5 IVs & & 1 \\
\hline HD 157170 & 7.97 & 3 & $\mathrm{kA} 0 \mathrm{hA} 1 \mathrm{~mA} 2 \mathrm{~V}$ & 68 & \\
\hline HD 157184 & 9.48 & 3 & A1 V & & \\
\hline HD 157389 & 9.98 & 3 & B9 IV-Vn & & 1 \\
\hline HD 158681 & 8.22 & 3 & B6 IV: & 69 & \\
\hline HD 158830 & 8.97 & 3 & A1 IV-V (shell) & 70 & 1 \\
\hline HD 159014 & 9.64 & 2 & B7 IV-V(e) & 71 & 1 \\
\hline HD 160461 & 7.51 & 3 & $\mathrm{~A} 1.5 \mathrm{IVn}$ & & \\
\hline HD 161576 & 9.26 & 3 & $\mathrm{~A} 4 \mathrm{Vn}$ & & \\
\hline HD 161595 & 9.17 & 3 & A1 Vas & & 1 \\
\hline HD 162220 & 6.66 & 3 & B9 IVn & & 1 \\
\hline HD 163296 & 6.85 & 1 & A 3 Vae kA1mA1 & 72 & 1 \\
\hline
\end{tabular}

52 Very deep H-line cores.

53 Great match to the standard star.

54 Very slight weakness in the Mg II 4481 line, and the Ca II K line is a little weak, but B9 is too early to claim weak metal lines.

55 Slightly shallow $\mathrm{H}$ cores.

56 Weak H cores.

57 Metal lines (except $\mathrm{K}$ line) are slightly weak. Not a $\lambda$ Boo star. $58 \mathrm{H}$ lines well-matched at A1 IV-V, not at A0 IV-V. But trace He suggests A0. Could alternatively be a low-luminosity late-B star, e.g. B9.5 Vbn. The Mg II 4481 line is weak, but rotation is very rapid, so probably not a $\lambda$ Boo star.

59 Very deep H-line cores.

60 Slightly shallow $\mathrm{H}$ cores.

61 Excellent match, except for slightly shallow $\mathrm{H}$ cores.

62 Not a $\lambda$ Boo star.

63 Sr lines are strong. The Mg II 4481 is slightly weak. Not a $\lambda$ Boo star.

64 Slight emission notch in $\mathrm{H} \beta$.

65 Classical shell star. Strong lines of Fe II, deep absorption cores in $\mathrm{H}$ lines, emission notch in $\mathrm{H} \beta$.

66 An extreme $\lambda$ Boo star.

67 A very marginal $\lambda$ Boo star.

68 Possibly a very mild and early Am star.

$69 \mathrm{H}$ cores are too deep for $\mathrm{B} 5 \mathrm{~V}$, and wings are too deep for B5 III. He lines are slightly weaker than B5, so B6 IV is the best match.

70 Fe II 4232 is slightly enhanced, as well as the one line of the Fe II (42) multiplet that is visible in this spectrum, suggesting a shell. $\mathrm{H}$ line cores are also quite deep, more so than can be explained by slow rotation.

71 Emission in core of $\mathrm{H} \beta$, infilling in $\mathrm{H} \gamma$. He I slightly weak for B7, so may be B7.5.

72 Not a $\lambda$ Boo star, since the Mg II 4481 line is normal.

73 Intermediate between the A9 Vn standard, 44 Cet, and the F0 V standard, HD23585, broadened to $v \sin i=150 \mathrm{~km} \mathrm{~s}^{-1}$.

74 Classic $\lambda$ Boo star.

75 Cores are too narrow for an earlier giant (e.g. A4 III-IV). The $\mathrm{Mg}$ II 4481 line is weak.

76 The CaI 4226 line is strong while Mg II 4481 is weak. A mild $\lambda$ Boo star.

77 The Mg II 4481 line is rather weak, but not much difference between $\mathrm{h}$ and $\mathrm{km}$ types. A mild $\lambda$ Boo star. This target was observed twice and each spectrum was classified independently, arriving at similar classifications. The Sr II 4077 line is unusually strong in one of the spectra, but the Sr II 4215 line is normal.

78 Metal weak, not clearly $\lambda$ Boo in nature.

79 Could serve as spectral standard for B9 Vbs.

80 Blue horizontal branch star? Mg II 4481 is weak, although not with respect to A5 II line ratios.

81 Emission in $\mathrm{H} \beta$ (in an inverted ' $\mathrm{w}$ ' shape), strong absorption in Si 4128-30 and the CaII K line.

82 Emission partially fills $\mathrm{H} \beta$.

83 Slightly noisy spectrum.

84 Possibly a mild Am star, with the $\mathrm{K}$ line weaker than the metals, but also definitely a rapid rotator.

85 Emission partially fills $\mathrm{H} \beta$.

86 Very rapid rotation. Even so, the Mg II 4481 line is weak.

87 Slightly shallow H cores. 
Table A1. continued. Spectral classes for the program stars.

\begin{tabular}{|c|c|c|c|c|c|}
\hline Obj Name & $\mathrm{V}$ mag & Group & Class & Note & IR \\
\hline HD 163921 & 9.52 & 3 & A9.5 Vn & 73 & 1 \\
\hline HD 168740 & 6.12 & 0 & A9 VskA2mA2 $\lambda$ Boo & 74 & 1 \\
\hline HD 168947 & 8.11 & 0 & A9 Vs kA3mA4 ( $\lambda$ Boo $)$ & 75 & \\
\hline HD 169142 & 8.16 & 3 & F1 VskA4mA5 ( $\lambda$ Boo $)$ & 76 & 1 \\
\hline HD 169346 & 9.27 & 3 & $\mathrm{~A} 8 \mathrm{VkA} 6 \mathrm{~mA} 6$ ( $\lambda$ Boo $)$ & 77 & \\
\hline HD 171013 & 8.6 & 3 & $\mathrm{~F} 2 \mathrm{VskA} 8 \mathrm{~mA} 7$ & 78 & 1 \\
\hline HD 176386 & 7.21 & 3 & B9 Vbs & 79 & 1 \\
\hline HD 176387 & 8.94 & 4 & A5 II-III kA0mA0 & 80 & 1 \\
\hline HD 184779 & 8.9 & 0 & F0 V kA5mA6 $(\lambda$ Boo $)$ & & \\
\hline HD 188230 & 8.18 & 3 & $\mathrm{~A} 0 \mathrm{Vbn}$ & & \\
\hline HD 261520 & 10.11 & 1 & B5 II-IIIe shell & 81 & 1 \\
\hline HD $288947 \mathrm{~A}$ & 11.13 & 2 & B7 IV-Ve & 82 & 1 \\
\hline HD 290469 & 9.87 & 3 & $\mathrm{~A} 4 \mathrm{Vs}$ & & 1 \\
\hline HD 290470 & 9.77 & 3 & $\mathrm{~A} 2.5 \mathrm{~V}$ & & 1 \\
\hline HD 290516 & 9.51 & 3 & B9 Vbn & & \\
\hline HD 290666 & 10.03 & 3 & A0 Van & & 1 \\
\hline HD 290684 & 9.7 & 3 & $\mathrm{~A} 2 \mathrm{VkA} 1$ & & \\
\hline HD 292895 & 11.16 & 2 & $\mathrm{~B} 8 \mathrm{Vn}$ & 83 & 1 \\
\hline HD 294054 & 9.6 & 3 & kA0hA0mA1 Vbn & 84 & \\
\hline HD 294103 & 9.7 & 3 & A1 Van & & 1 \\
\hline HD 294253 & 9.67 & 3 & A0 Va kB8.5mB9 $(\lambda \mathrm{Boo})$ & & 1 \\
\hline HD 296192 & 10.21 & 2 & B7.5 IV-Ve & 85 & 1 \\
\hline HD 304838 & 9.87 & 3 & $\mathrm{~B} 8.5 \mathrm{~V}$ & & \\
\hline HD 307860 & 8.28 & 3 & $\mathrm{~B} 8.5 \mathrm{Vnn}$ & & 1 \\
\hline HD 308889 & 10.64 & 2 & B6 V(e) & & 1 \\
\hline HD 309344 & 10.85 & 2 & A4 III-IV & & 1 \\
\hline HD 314915 & 11.31 & 2 & A9 VnkA5mA5 ( $\lambda$ Boo $)$ & 86 & 1 \\
\hline HD 318093 & 9.71 & 3 & $\mathrm{~A} 0 \mathrm{Va}+\mathrm{s}$ & & 1 \\
\hline HD 318099 & 9.86 & 3 & A0 Van & 87 & \\
\hline HD 318127 & 9.82 & 3 & A1 IVn & & 1 \\
\hline HD 320460 & 10.63 & 2 & B5 IIIe & 88 & 1 \\
\hline HD 320765 & 8.73 & 3 & $\mathrm{~A} 1 \mathrm{~V}$ & & 1 \\
\hline HD 322663 & 9.77 & 3 & A1 IVn & & 1 \\
\hline $\mathrm{BD}+002757$ & 10.64 & $\mathrm{~K} 2$ & $\mathrm{~F} 5 \mathrm{~V}: \mathrm{mF} 2 \mathrm{gF} 5$ & & \\
\hline BD-02 2182 & 9.71 & 2 & $\mathrm{~B} 2 \mathrm{Vn}$ & & 1 \\
\hline BD-08 1151 & 9.82 & 3 & A2 IV-V & & 1 \\
\hline BD-11 1239 & 9.7 & 3 & A7 VkA3mA3 $((\lambda$ Boo $))$ & & 1 \\
\hline BD-11 1762 & 10.05 & 3 & B2 IV-Vn & & 1 \\
\hline BD-15 1548 & 9.88 & 1 & B7 IIIe He-wk & & 1 \\
\hline BD-15 4515 & 9.97 & 4 & $\mathrm{~F} 2 \mathrm{VkA} 4 \mathrm{~mA} 6 \lambda \mathrm{Boo}$ & & 1 \\
\hline CD-31 4428 & 9.86 & 3 & A2 Van & & \\
\hline CD-37 3833 & 9.92 & 3 & $\mathrm{~A} 2 \mathrm{VnkA} 0$ & & 1 \\
\hline CD-48 3541 & 9.67 & 3 & $\mathrm{~A} 2 \mathrm{VnkA} 0 \mathrm{~mA} 1$ & 89 & 1 \\
\hline CD-55 2595 & 10.31 & 2 & $\mathrm{~B} 1 \mathrm{Ve}$ & 90 & 1 \\
\hline CD-58 3782 & 9.79 & 3 & A3 Van & & \\
\hline CD-59 1764 & 9.65 & 3 & $\mathrm{~A} 0.5 \mathrm{~V}$ & & 1 \\
\hline CD-60 1932 & 9.93 & 3 & A0 Vnn & 91 & 1 \\
\hline CD-60 1956 & 9.65 & 3 & $\mathrm{~A} 0.5 \mathrm{~V}$ & & \\
\hline CD-60 1986 & 9.57 & 3 & A2 Van & & \\
\hline CD-60 4157 & 9.38 & 3 & A1 Van & & 1 \\
\hline CD-60 6017 & 9.63 & 3 & A8IV-V & & \\
\hline CD-60 6021 & 9.73 & 3 & B7 IVn & & \\
\hline CPD-20 1613 & 10.0 & 3 & A0.5 V kB9.5 & & \\
\hline CPD-583071 & 9.85 & 3 & A3 Va & & \\
\hline CPD-583106 & 9.76 & 3 & $\mathrm{~A} 1.5 \mathrm{Vn}$ & & \\
\hline CPD-583138 & 9.73 & 3 & $\mathrm{~A} 1.5 \mathrm{Vs}$ & & 1 \\
\hline IK Hya & 10.23 & 4 & B7 II kA3mA3 & 92 & \\
\hline KK Oph & 10.99 & 1 & A9:e & 93 & 1 \\
\hline NGC 638322 & 12.49 & 1 & $\mathrm{~F} 1 \mathrm{~V}(\mathrm{e}) \mathrm{kA} 6 \mathrm{~mA} 6 \lambda$ Boo? & 94 & 1 \\
\hline T Ori & 11.25 & 1 & A 8 Vne kA1mA2 $\lambda$ Boo & 95 & 1 \\
\hline V1012 Ori & & 1 & F3 V kA5n composite & 96 & \\
\hline V748 Cen & 11.93 & 2 & $\mathrm{~A} 0 \mathrm{e}$ & 97 & 1 \\
\hline V Lep & 9.71 & 3 & $\mathrm{~F} 0.5 \mathrm{~V}(\mathrm{n}) \mathrm{kA} 8 \mathrm{~mA} 9$ & 98 & 1 \\
\hline
\end{tabular}

\footnotetext{
88 Emission core in $\mathrm{H} \beta$, infilling in $\mathrm{H} \gamma$, and possibly $\mathrm{H} \delta$.

89 Not a $\lambda$ Boo star, just slightly metal weak

90 Spectrum contaminated, $\mathrm{H} \beta$ partially filled with emission.

91 Rotating more rapidly than the high $v \sin i \mathrm{~A} 0 \mathrm{~V}$ standard.

92 Known RRLyr variable.

93 Emission in $\mathrm{H} \beta$, Ca II $\mathrm{K}$ and $\mathrm{H}$, and some other metallic lines.
}

\footnotetext{
Probably pre main sequence, or possibly an RS CVn variable. This is KK Oph, a known Herbig Ae/Be star.

94 The Ca II K line has a peculiar profile (broad but shallow). Enhanced G-band absorption suggests this is a composite spectrum. The Mg II 4481 line is slightly weak. A high-resolution spectrum is needed to determine if this is a mild $\lambda$ Boo star or a composite instead.

${ }^{95} \mathrm{H} \beta$ in emission, emission core in $\mathrm{H} \gamma$.

96 Might be a triple system: both line veiling, indicating a hotter companion, and a strong G-band red edge, indicating a cooler companion, are evident.

97 Most lines are in emission, $\mathrm{H} \beta$ strongly so. SpT from Ca II K line.

98 Not a $\lambda$ Boo star, just slightly metal weak.
} 